\title{
Journal of the Saudi Heart Association
}

\section{latrogenic withdrawal syndrome in the pediatric cardiac intensive care unit: Incidence, risk factors and outcome}

Follow this and additional works at: https://www.j-saudi-heart.com/jsha

Part of the Cardiology Commons (c) (i) (8)

This work is licensed under a Creative Commons Attribution-Noncommercial-No Derivative Works 4.0 License.

\section{Recommended Citation}

Habib, Eiad; Almakadma, Abdul Hakim; Albarazi, Mohieddin; Jaimon, Somiya; Almehizia, Rayd; Al Wadai, Abdullah; and Abouelella, Raja (2021) "latrogenic withdrawal syndrome in the pediatric cardiac intensive care unit: Incidence, risk factors and outcome," Journal of the Saudi Heart Association: Vol. 33 : Iss. 4 , Article 1.

Available at: https://doi.org/10.37616/2212-5043.1268

This Original Article is brought to you for free and open access by Journal of the Saudi Heart Association. It has been accepted for inclusion in Journal of the Saudi Heart Association by an authorized editor of Journal of the Saudi Heart Association. 


\title{
Iatrogenic Withdrawal Syndrome in the Pediatric Cardiac Intensive Care Unit: Incidence, Risk Factors and Outcome
}

\author{
Eiad Habib ${ }^{a} *$, Abdul Hakim Almakadma ${ }^{a}$, Mohieddin Albarazi ${ }^{a}$, Somiya Jaimon ${ }^{\text {, }}$ \\ Rayd Almehizia $^{\mathrm{b}}$, Abdullah Al Wadai ${ }^{\mathrm{b}}$, Raja Abouelella ${ }^{\mathrm{b}}$

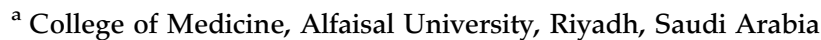 \\ ${ }^{\mathrm{b}}$ Pediatric Cardiac Surgical Intensive Care Unit, King Faisal Specialist Hospital and Research Center, Riyadh, Saudi Arabia
}

\section{Abstract}

Objectives: Critically ill children and neonates routinely receive opioids and benzodiazepines for analgesia and sedation in the pediatric intensive care unit (PICU). Prolonged opioid therapy often leads to tolerance and is associated with iatrogenic withdrawal syndrome (IWS) when opioids and benzodiazepines are weaned or discontinued. Our aim is to study the incidence, risk factors and outcome of IWS following use of a withdrawal protocol on pediatric patients after cardiac surgery.

Methods: We prospectively included all postoperative pediatric patients who developed IWS after being assessed by the Withdrawal Assessment Tool (WAT-1) score (WAT-1 $\geq 3$ ) over 9 months. Unit-based withdrawal management protocol was used. Patients' outcome was assessed by duration to be completely off opioids \& benzodiazepines, duration of ICU and hospital stay.

Results: 432 patients were admitted during study period. Twenty-five patients $(5.8 \%)$ developed IWS. The mean age and weight was $15.2 \pm 31.4$ months and $6.9 \pm 7.7 \mathrm{~kg}$ respectively. $60 \%$ of the patients who developed withdrawal had previous ICU admissions with exposure to sedatives. In the IWS group the mean duration for use of fentanyl and midazolam infusions was $8.6 \pm 5.1 \& 9.6 \pm 6$ days respectively. The mean duration for completely stopping opioids and benzodiazepines was $17.2 \pm 6.8$ days with a mean duration of ventilation of $8.2 \pm 6.8$ days. The mean ICU and hospital stay for IWS patients was $26.5 \pm 21.9$ and $31.5 \pm 21.1$ days respectively. There was statistically significant difference in the mean age, and duration of use of sedatives between the patients who developed IWS and those who did not with Pvalue of 0.003 and $<0.0001$, respectively.

Conclusion: Withdrawal syndrome after pediatric cardiac surgery prolongs ICU and hospital stay. Our data shows that younger age and longer duration for use of sedatives seem to be risk factors for developing withdrawal syndrome.

Keywords: Iatrogenic withdrawal, Pediatric cardiac intensive care, Incidence, Outcome, Middle East

\section{Introduction}

I n the pediatric critical care setting, children and neonates routinely receive opioids and benzodiazepines for analgesia and sedation to reduce pain, anxiety and stress responses; retain monitoring devices; facilitate ventilation; and avoid secondary complications.
Prolonged opioid and benzodiazepine therapy often leads to tolerance, seen as diminishing pharmacologic effects, and is associated with withdrawal symptoms when discontinued. A syndrome observed in many of these pediatric patients is Iatrogenic Withdrawal Syndrome (IWS). IWS has been observed to have a sudden appearance in patients exposed to opioids for a prolonged period; although

Received 14 June 2021; revised 23 August 2021; accepted 29 August 2021.

Available online 15 October 2021

* Corresponding author at:

E-mail address: ehabib@alfaisal.edu (E. Habib). 
it can be seen as short as three days, or at large cumulative doses once treatment is discontinued abruptly. IWS is responsible for prolonging the hospitalization period, the Pediatric Intensive Care Unit (PICU) stay as well as mechanical ventilation duration $[1,2]$.

Studies suggest that IWS occurs in $35-64 \%$ of patients admitted to PICU [1,3]. A recent clinical report by the American Academy of Pediatrics (AAP) predicts that the likelihood of developing withdrawal syndrome in patients exposed to 7 days of fentanyl therapy or a threshold at $2 \mathrm{mg} / \mathrm{kg}$ of fentanyl exposure, is likely to fall between 50 and $100 \%$ [4].

Knowledge of possible risk factors for the development of IWS allows healthcare providers to identify patients at risk. Published studies cite a diverse array of information describing incidences and influencing factors for the development of withdrawal symptoms after long use of benzodiazepines or opioids in children [1,5,6]. Known risk factors associated with IWS include the cumulative dose of opioid or benzodiazepine administered, the duration of treatment with these analgesics or sedatives, regular exposure for $72 \mathrm{~h}$ or longer, younger age and the duration of extracorporeal membrane oxygenation (ECMO). Other risk factors include cognitive impairment [7].

Lack of a unified weaning protocol for withdrawal assessment and standardized practices with clear guidelines are crucial missing steps that, if implemented correctly, can drastically reduce the prevalence of IWS. Therefore, a unit-based withdrawal management protocol was formulated in our study and approved for use in the cardiac surgical intensive care unit (CSICU) for children.

Information about incidence and risk factors for sedation withdrawal in pediatric cardiac ICU patients is limited, especially in the Middle East region. Therefore, we decided to prospectively study the incidence, possible risk factors and outcomes of using our newly developed unit-based withdrawal management protocol on this very special patient population. To our knowledge, this was the first prospective study about withdrawal syndrome conducted in the Middle East.

\section{Materials and methods}

We conducted a non-randomized, prospective, single-center observational study between December 2016 and September 2017. Our pediatric CSICU has
Abbreviations
PICU Pediatric intensive care unit
WAT-1 Withdrawal Assessment Tool
IWS iatrogenic withdrawal syndrome
ECMO extracorporeal membrane oxygenation
CSICU cardiac surgical intensive care unit
RACHS Risk Adjustment for Congenital Heart Surgery
IQR

12 beds and cares for a variety of congenital cardiac surgery and heart transplant patients. Ethical approval was obtained from the Institutional Review Board (IRB) committee at our hospital.

As per our unit pain and sedation management protocol, all patients received fentanyl infusions and intravenous (IV) paracetamol for analgesia until they were extubated and midazolam infusions for sedation as needed.

We used the Withdrawal Assessment Tool (WAT-1), which is a validated assessment tool for monitoring withdrawal symptoms in pediatric patients. The assessment tool consists of 11 items, which are determined by the following components: a review of the patient's records for the past $12 \mathrm{~h}$, a direct observation of the patient for $2 \mathrm{~min}$, a patient assessment using a progressive stimulus and an assessment of post-stimulus recovery [2]. Scores range from 0 to 12 and a score $\geq 3$ indicates the presence of signs or symptoms of IWS. As defined by the WAT-1 official definition, the severity of IWS is higher as the score increases.

In our study, WAT-1 assessment was started on the first day of weaning patients who were ready to commence a weaning plan and who had received opioids and/or benzodiazepines by infusion, intermittent IV dosing or oral dosing for prolonged periods (e.g. 5 days or more) or those who showed clinical signs of IWS. A baseline withdrawal score using the WAT-1 before any of the drugs are weaned was obtained. Scores were done every $12 \mathrm{~h}$ at 06:00 and 18:00 $\mathrm{h}$ - based on bedside staff clinical judgement, the need for more frequent assessment was done - increased to every $4-8 \mathrm{~h}(\mathrm{~h})$ if withdrawal scores were high and intervention was required. IWS was diagnosed when WAT-1 scores were $\geq 3$ on 2 consecutive assessments and after exclusion of pain or other pathological factors then the unit-based protocol was used as a guideline to direct the weaning plan (Appendix I). Morphine and lorazepam were our choices for the enteral 
substitutes of IV fentanyl and midazolam. Use of dexmedetomidine as an adjuvant to facilitate weaning was left to the intensivist choice and bedside evaluation. Scoring was continued for $48 \mathrm{~h}$ after the last opioid/benzodiazepine was administered (Appendix II).

Inclusion criteria for enrollment was defined as all pediatric patients under 18 years of age with heart disease admitted to CSICU after cardiac surgery who developed IWS after being assessed by WAT-1 score and required to be on sedation weaning plan (as per unit protocol). Exclusion criteria was defined as patients who did not have IWS after being assessed by WAT-1 score and patients with known neurological disorders or complications, e.g. seizure disorders.

In addition to collection of demographic variables such as age, gender, and weight, we also included potential risk factors including number of ICU days at diagnosis of IWS, previous ICU admissions, complexity of surgery done using Risk Adjustment for Congenital Heart Surgery (RACHS) score and the total duration they were on opioids and benzodiazepines.

Primary outcome was defined as the time taken for patients to fully recover from IWS as evidenced by being completely off opioids and benzodiazepines and WAT-1 score $<3$ for $72 \mathrm{~h}$ following cessation of all medications. Secondary outcomes were defined as mean duration of mechanical ventilation as well as ICU and hospital stays, and were compared between the group who developed IWS and those who didn't during the same study period.

For statistical analysis, the SPSS version 19.0 statistical package was used to analyze the results. Statistical significance was defined as $P<0.05$. The statistical analyses were primarily focused on estimation and for this purpose $95 \%$ confidence intervals for incidence, mean time to wean-off, mean time to ICU discharge, etc. were calculated. Chi-square test was carried out for categorical variables.

\section{Results}

432 patients were admitted during study period. 25 patients $(5.8 \%)$ developed IWS. Of those, $13(52 \%)$ were males and $12(48 \%)$ were females.

The mean age and weight for patients with IWS was $15.2 \pm 31.4$ months and $6.9 \pm 7.7 \mathrm{~kg}$ respectively. The median age and weight for patients with IWS was 4.5 months (IQR 9.75 months) and $4.25 \mathrm{~kg}$ (IQR $3.15 \mathrm{~kg}$ ) respectively. Age and duration of use of fentanyl and midazolam were significantly different between the group who developed IWS and those who did not (Table 1). The mean WAT-1 score at the
Table 1. Prominent risk factors seen in our admitted patients with confirmed IWS.

\begin{tabular}{|c|c|c|c|}
\hline Factor & $\begin{array}{l}\text { IWS group } \\
(\mathrm{N}=25)\end{array}$ & $\begin{array}{l}\text { Non-IWS } \\
\text { group } \\
(\mathrm{N}=407)\end{array}$ & P-value \\
\hline Age (months) & $15.2 \pm 31.4$ & $35.2 \pm 45.1$ & 0.003 \\
\hline Weight (kg) & $6.9 \pm 7.7$ & $9.8 \pm 8.4$ & 0.09 \\
\hline $\begin{array}{l}\text { Mean duration of } \\
\text { fentanyl (days) }\end{array}$ & $8.6 \pm 5.1$ & $3.2 \pm 2.1$ & $<0.0001$ \\
\hline $\begin{array}{l}\text { Mean duration of } \\
\text { midazolam (days) }\end{array}$ & $9.7 \pm 6.1$ & $2 \pm 1$ & $<0.0001$ \\
\hline
\end{tabular}

time of diagnosis was 6 , with a $95 \%$ confidence interval of 0.71 . Fifteen patients $(60 \%)$ in the IWS group had a previous history of ICU admissions and $13(52 \%)$ of patients with IWS were in RACHS 4 category (Fig. 1). The mean duration for use of fentanyl and midazolam infusions in the IWS group was $8.6 \pm 5.1$ days and $9.7 \pm 6.1$ days respectively while the mean duration of oral morphine and lorazepam use was $8.6 \pm 3.4$ days.

The mean cumulative doses of fentanyl and midazolam in the IWS group were $10.08 \mathrm{mg}$ and $504 \mathrm{mg}$, respectively. The mean cumulative doses of fentanyl and midazolam in the non-IWS group were $3.84 \mathrm{mg}$ and $48 \mathrm{mg}$, respectively. Withdrawal management algorithms (Appendix I \& II) were used for all patients included in the study. One patient $(4 \%)$ was

\section{Percentage of patients with IWS}

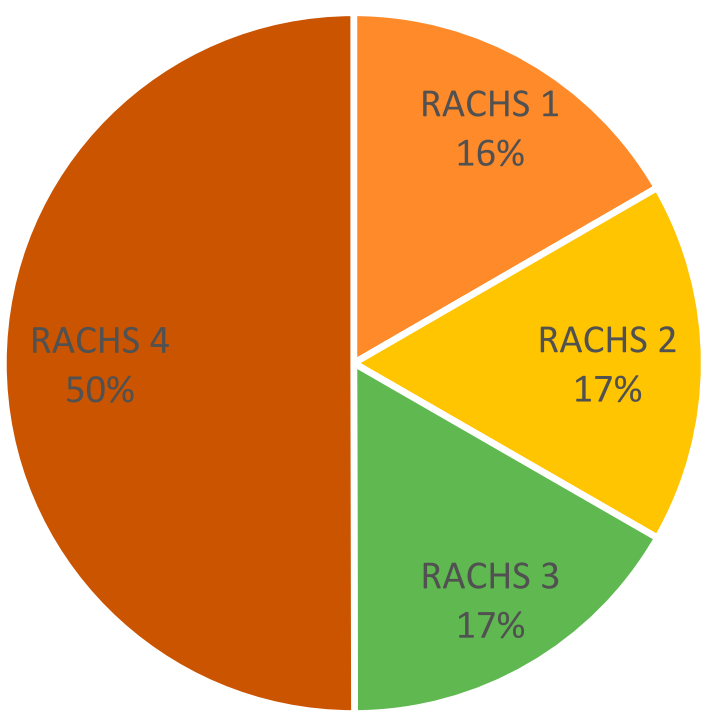

- RACHS 1 RACHS 2 RACHS 3 RACHS 4

Fig. 1. Risk adjustment for congenital heart surgery and its relation to our patients with latrogenic withdrawal syndrome. 
Table 2. ICU days at diagnosis for patients with confirmed IWS.

\begin{tabular}{ll}
\hline Category & Number of patients $(\%)$ \\
\hline$<4$ days & $1(4 \%)$ \\
$4-10$ days & $18(72 \%)$ \\
$>10$ days & $6(24 \%)$ \\
Median ICU days at diagnosis & 7 \\
\hline
\end{tabular}

Table 3. Outcomes for patients with confirmed IWS.

\begin{tabular}{ll}
\hline Item & Mean \pm SD (days) \\
\hline Duration for completely stopping & $17.2 \pm 6.8$ \\
$\quad$ opioids and benzodiazepines & \\
Duration of ventilation & $8.2 \pm 6.8$ \\
ICU stay & $26.5 \pm 21.9$ \\
Hospital stay & $31.5 \pm 21.1$ \\
\hline
\end{tabular}

diagnosed with IWS at 3 days following ICU admission and $72 \%$ had IWS between 4 and 10 days of ICU stay (Table 2). Dexmedetomidine was used for 10 patients $(40 \%)$ with median duration of 1 day (range 1-5 days). The average number of days for completely stopping opioids and benzodiazepines (both IV and oral) in the IWS group was $17.2 \pm 6.8$ days (Table 3). None of our patients was discharged home on opioids or benzodiazepines. The duration of ventilation was $8.2 \pm 6.8$ days in the IWS group in comparison to $1.3 \pm 3$ days in the non-IWS group ( $p<0.0001)$. Median duration of ventilation in IWS group was 7 days (IQR 4.25 days). The median hospital stay for IWS group was 20 days (IQR 17 days). There was a statistically significant difference between ICU and hospital stays between IWS and non-IWS groups with a P-value of $<0.0001$ (Fig. 2).

Figure 1 illustrates our patients with Iatrogenic Withdrawal Syndrome in relation to the Risk
Adjustment for Congenital Heart Surgery (RACHS). The figure demonstrates that patients with RACHS score 4 represented $50 \%$ of our patients with IWS, while RACHS scores of 1, 2, and 3 combined made up the other $50 \%$ of patients. A Chi-square test done demonstrated that patients with a RACHS score of 4 have a statistically significant risk of developing IWS $(\mathrm{p}=0.046)$.

Table 1 demonstrates some of the variables we assessed as potential risk factors for developing IWS in our study. These risk factors include age, weight, as well as mean durations of fentanyl and midazolam use. Infants who developed IWS had a mean age of $15.2 \pm 31.4$ months, which was significantly lower than infants in the non-IWS group at $35.2 \pm 45.1$ months ( $p$-value $=0.003$ ). There was no statistically significant difference in the weight between the 2 groups $(6.9 \pm 7.7 \mathrm{~kg}$ vs $9.8 \pm 8.4 \mathrm{~kg})$. The mean duration of fentanyl use was significantly higher in the IWS group ( $8.6 \pm 5.1$ days $)$ as opposed to the non-IWS group ( $3.2 \pm 2.1$ days), giving a $\mathrm{p}$ value of $<0.0001$. In addition, the mean duration of midazolam use was also significantly higher in the IWS group (9.7 \pm 6.1 days) as opposed to the nonIWS group ( $2 \pm 1$ days), giving a p-value of $<0.0001$.

Table 2 demonstrates the number of ICU days at diagnosis for patients with confirmed IWS. 18 patients $(72 \%)$ were diagnosed with IWS between 4 and 10 days of ICU admission. 6 patients $(24 \%)$ were diagnosed more than 10 days after admission, and only 1 patient $(4 \%)$ was diagnosed at 3 days following ICU admission. The median ICU days at diagnosis for all patients was 7 .

Table 3 demonstrates the outcomes for patients with confirmed IWS in our study. The mean number of days for completely stopping opioids and benzodiazepines was $17.2 \pm 6.8$ days. The mean

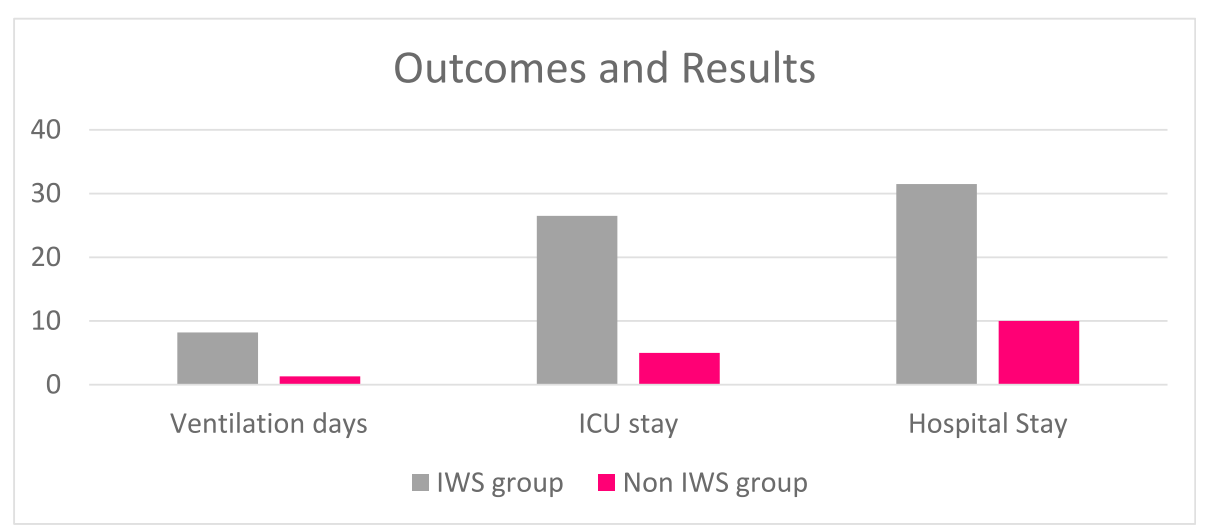

Fig. 2. Comparison of outcomes and results in all patients with confirmed IWS vs non-IWS group. $p<0.0001$ for all subgroups. 
number of days patients were on mechanical ventilation was $8.2 \pm 6.8$ days. The mean number of days for the ICU stay was $26.5 \pm 21.9$ days. Lastly, the mean number of days for total hospital stay was $31.5 \pm 21.1$ days.

Figure 2 illustrates the outcomes for patients with confirmed IWS in comparison to the non-IWS group. The mean number of days for the duration of ventilation was significantly higher in the IWS group $(8.2 \pm 6.8$ days) in comparison to $1.3 \pm 3$ days in the non-IWS group ( $\mathrm{p}<0.0001)$. The mean number of days for the ICU stay was $26.5 \pm 21.9$ days in the IWS group as opposed to $5 \pm 5$ days in the non-IWS group ( $p<0.0001)$. Lastly, the mean number of days for total hospital stay was also significantly higher in the IWS group $(31.5 \pm 21.1$ days) in comparison to $10 \pm 9$ days in the non-IWS group ( $\mathrm{p}<0.0001)$.

\section{Discussion}

The incidence of IWS is variable in the literature, with numbers ranging from $35 \%$ to $64 \%[1,3]$. In an observational multicenter study done in 2017 by Amigoni et al., withdrawal syndrome was recognized in $64 \%$ of PICU patients $(n=113)$ who received sedation and analgesia for at least five days [3]. There is a lack in universal guidelines of clinical practice in preventing IWS in critically ill patients, which may explain the variable prevalence rates of the disease. Further complicating matters are the unclear signs and symptoms of IWS. The signs and symptoms caused by IWS as a result of overmedication overlap with the signs and symptoms of agitation caused by mechanical ventilation, illness and inadequate pain medication [4].

Patients with heart diseases admitted to PICU after cardiac surgery are a special category of patients. Many of them require multiple PICU admissions during their lifetimes and get exposed to opioids and benzodiazepines for multiple reasons, consequently predisposing them to IWS. However, the incidence of IWS in this group of patients is poorly studied. We could not appreciate any studies commenting on this group in the literature, but it is known that patients with comorbidities are more likely to develop IWS, as reduced clearance of medications may occur due to organ failure or inflammation [8]. This is why we aimed to look at our local incidence, especially since this study was done a couple of years following our application of a strict pain and sedation management protocol in our pediatric cardiac surgical ICU [9].

We reported an incidence of $5.8 \%$, which is significantly less than what has been previously reported in literature. This may be due to the fact that using the pain management protocol has alerted us for using appropriate doses over a shorter duration. Furthermore, we began to monitor for IWS after a relatively short period ( 3 days) of continuous benzodiazepine/ opioid therapy. The use of a standardized rescue protocol is imperative in reducing the duration of therapy and IWS symptoms [4]. Therefore, we speculate that our lower IWS incidence could be due to a strict pain and sedation regimen and the use of a standardized assessment and weaning protocol in addition to regular education and raising team awareness regarding the assessments and consequences of using pain medications and IWS.

The cohort of patients studied was representative of the normal pediatric cardiac surgery ICU in terms of age range, gender distribution, and types of diagnoses. However, the mean age for infants in the IWS group was significantly lower than infants in the non-IWS group. This finding is similar to that reported by Best et al., in 2017 [10], which concluded that children younger than 6 months are most likely to develop IWS. Even without any renal or hepatic dysfunction, the decreased metabolic and clearance rates of analgesics or sedatives results in increased half-lives and serum concentrations and ultimately increased receptor occupancy [10].

Treatment for 5 or more days with opioids or benzodiazepines is widely considered to be a risk factor for the development of IWS [11]. In our population, $72 \%$ of patients were considered high risk for withdrawal based upon this risk factor.

There is evidence that using an IWS management protocol decreases the incidence of IWS [4,12]. In this study, we developed our own unit based protocol which was slightly different in the rate of weaning being a bit faster. However, that has worked well for us and led to shorter duration on both IV \& enteral medications in addition to less hospital stay days in comparison to that reported by Amirnovin et al. [12]. In addition, this group reported that $6 \%$ of their patients were discharged home on opioids, while our cohort did not have any. We believe that individualization of weaning plans according to the patient's baseline WAT-1 score and having more frequent follow up assessments has helped as well. 
Several reviews proposed that drugs such as clonidine, dexmedetomidine, or methadone could play a role in reduction of IWS severity in pediatric patients, but there was no conclusive data reported in literature $[11,13,14]$. Given the limited evidence, their best assessment concluded that adjuvant drugs should be used with caution. While formulating our unit-based withdrawal protocol, we discussed the doses and regimens with a pediatric cardiac surgery ICU clinical pharmacist and subsequently based our exact doses and stepwise dose adjustment on the assessment and medication availability at our institute. Since enteral clonidine was not available at our hospital, it was not included in the protocol while dexmedetomidine was used in selected cases based on clinician assessment for its suitability and effect on hemodynamics.

Finally, we found significant differences in duration of mechanical ventilation; ICU and hospital stay between patients with or without IWS. These findings are well-documented in the literature [2,3,15]. On further stratification of our cohort according to the Risk Adjustment for Congenital Heart Surgery (RACHS) score, which is a validated scoring system indicating the severity of congenital cardiac surgery, a statistically significant proportion of our patients had a RACHS score of 4 . According to the literature, a higher RACHS score correlates to increased duration on pressors, increased duration of mechanical ventilation, increased ICU and hospital stays as well as increased mortality [16-18]. However, the distinction between whether it is the patients' conditions that best predict these outcomes, concomitantly necessitating prolonged analgesic and sedative treatment, versus the analgesic and sedative treatment along with the weaning protocols being the culprits for prolonged mechanical ventilation and length of stay independent of the patients' conditions, is yet to be clarified. Further research to study these concerns and identify preventative strategies with the aim of reducing iatrogenic harm to patients is needed.

\subsection{Limitations}

This is a single center, non-randomized observational study with a small number of patients. The study did not assess risk factors at the level of the system \& process e.g. protocol adherence, and providers' ability to maintain a consistent weaning plan in some children. Moreover, although the WAT-1 has a sensitivity of $87 \%$ and specificity of $88 \%$, it has been shown to be more likely to detect withdrawal symptoms of opioids than benzodiazepines. To date, however, there are no assessment tools that can differentiate withdrawal from opioids or withdrawal from benzodiazepines $[4,19]$. In addition, the concomitant administration of opioids and benzodiazepines limits the interpretation of our findings; however, this sedation regimen reflects the current clinical practice. Finally, the association between IWS and end-organ impairment and its effect on medication clearance was not explored in our study, and this can potentially be explored further later on.

\section{Conclusion}

Iatrogenic Withdrawal syndrome after pediatric cardiac surgery prolongs ICU and hospital stay.

Our data suggests that younger age, complexity of cardiac surgeries and longer duration for use of sedatives seems to increase the risk for developing withdrawal syndrome. Use of withdrawal management protocols is helpful in the management, but prevention whenever possible will be better. Given the state of existing evidence, well-designed prospective studies are required to better characterize IWS in critically ill pediatric cardiac patients. The introduction of a formalized assessment protocol is fundamental in preventing IWS and the use of an opioid weaning program can improve consistency of care and help avoid complications and increased hospital stays. 


\section{Author contributions}

Conception and design of Study: Eiad Habib, Abdul Hakim AlMakadma, Mohieddin Albarazi, Abdullah Al Wadai, Raja Abouelella. Literature review: Eiad Habib, Abdul Hakim AlMakadma, Mohieddin Albarazi. Acquisition of data: Eiad Habib, Abdul Hakim AlMakadma, Mohieddin Albarazi, Somiya Jaimon, Rayd Almehizia. Analysis and interpretation of data: Eiad Habib, Abdul Hakim AlMakadma, Mohieddin Albarazi. Research investigation and analysis: Eiad Habib, Abdul Hakim AlMakadma, Mohieddin Albarazi, Somiya Jaimon, Rayd Almehizia, Abdullah Al Wadai, Raja Abouelella. Data collection: Eiad Habib, Abdul Hakim AlMakadma, Mohieddin Albarazi, Somiya Jaimon, Rayd Almehizia. Drafting of manuscript: Eiad Habib, Abdul Hakim AlMakadma, Mohieddin Albarazi, Somiya Jaimon, Rayd Almehizia, Abdullah Al Wadai, Raja Abouelella. Revising and editing the manuscript critically for important intellectual contents: Eiad Habib, Abdul Hakim AlMakadma, Mohieddin Albarazi, Somiya Jaimon, Rayd
Almehizia, Abdullah Al Wadai, Raja Abouelella. Data preparation and presentation: Eiad Habib, Abdul Hakim AlMakadma, Mohieddin Albarazi. Supervision of the research: Abdullah Al Wadai, Raja Abouelella. Research coordination and management: Rayd Almehizia, Abdullah Al Wadai, Raja Abouelella.

\section{Acknowledgements}

We would like to thank Mr. Yousef Daibes and Ms. Victoria Smith for their valuable efforts during bedside assessments and in data collection.

\section{Disclosure of funding}

This research did not receive any specific grant from funding agencies in the public, commercial, or not-for-profit sectors.

\section{Conflict of interest}

None declared. 


\section{Appendix I. Algorithm for Weaning in Combined Opioids and Benzodiazepines}

\section{Algorithm for Weaning Combined Opioids and Benzodiazepines}

\section{Opioids/Benzodiazepines 5-10 days}

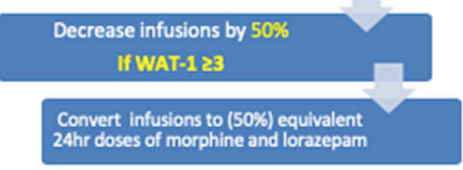

Opioids/Benzodiazepines $>10$ days

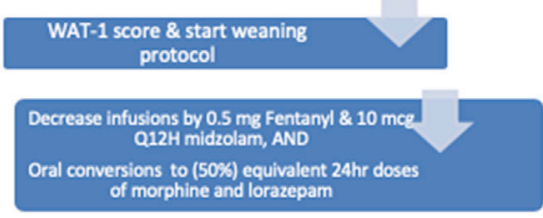

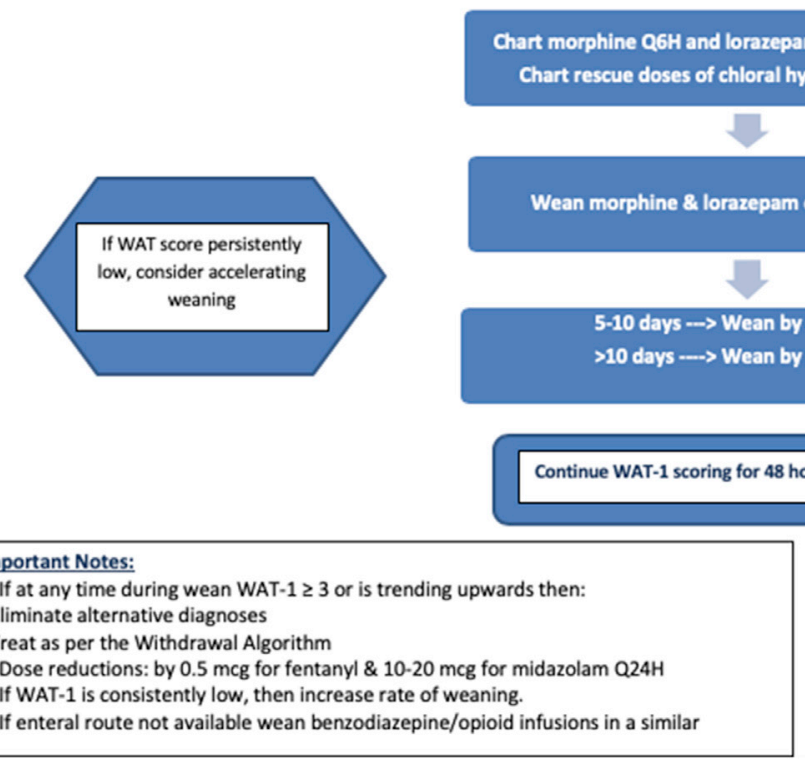




\section{Appendix II. Algorithm for Withdrawal Management.}

\section{Withdrawal Algorithm}

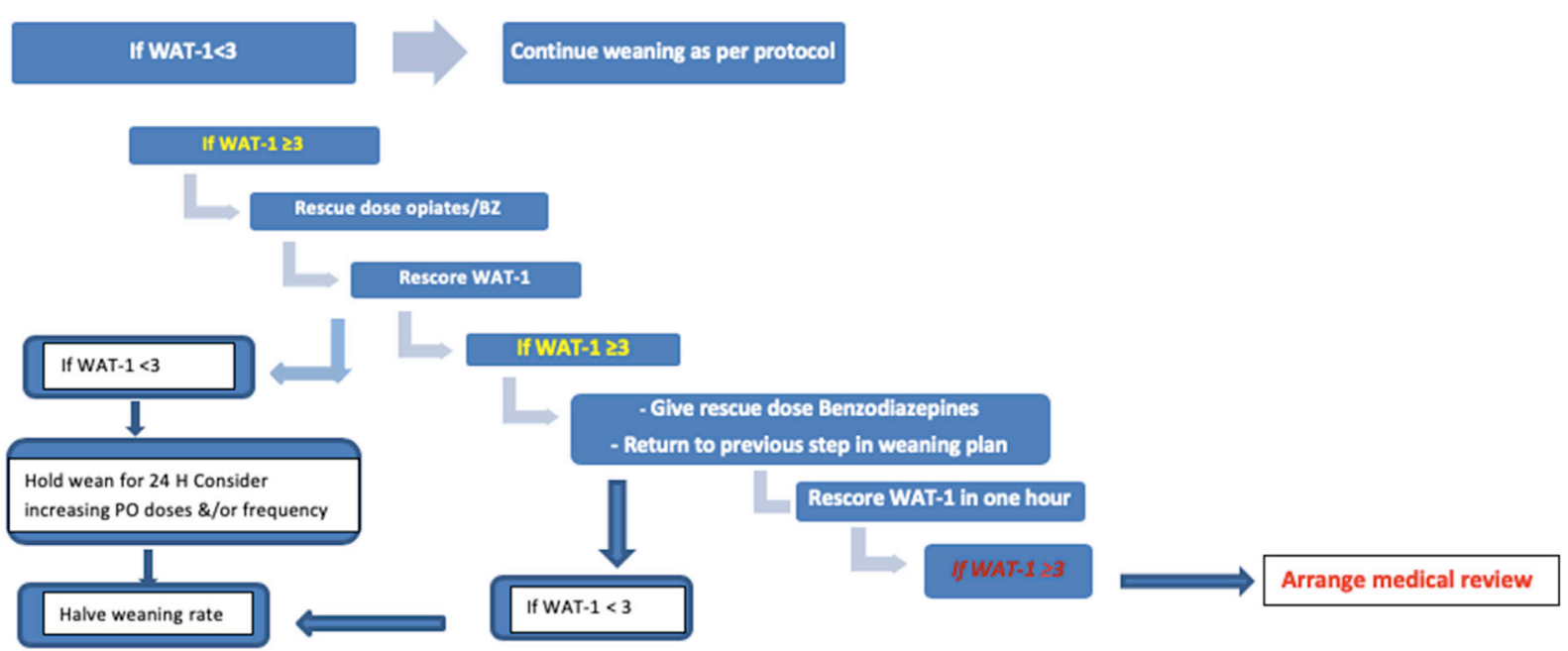

Important Notes:

1- If at any time during wean WAT- $1 \geq 3$ or is trending upwards then: Eliminate alternative diagnoses

2- Increase Morphine by $0.05 \mathrm{mg} / \mathrm{kg} /$ dose

3- Lorazepam: $1^{\text {st }}$ increase to $\mathrm{Q} 6 \mathrm{H}$, then increase by $0.01 \mathrm{mg} / \mathrm{kg} /$ dose 


\section{References}

[1] Da Silva PSL, Reis ME, Fonseca TSM, Fonseca MCM. Opioid and benzodiazepine withdrawal syndrome in PICU patients: which risk factors matter? J Addiction Med 2016;10:110-6. https://doi.org/10.1097/ADM.0000000000000197.

[2] Franck LS, Harris SK, Soetenga DJ, Amling JK, Curley MAQ. The Withdrawal Assessment Tool-1 (WAT-1): an assessment instrument for monitoring opioid and benzodiazepine withdrawal symptoms in pediatric patients. Pediatr Crit Care Med 2008;9:573-80. https://doi.org/10.1097/PCC.0b013e31818c8328.

[3] Amigoni A, Mondardini MC, Vittadello I, Zaglia F, Rossetti E, Vitale F, et al. Withdrawal assessment tool-1 monitoring in PICU: a multicenter study on iatrogenic withdrawal syndrome. Pediatr Crit Care Med 2017;18: e86-91. https://doi.org/10.1097/PCC.0000000000001054.

[4] Cramton REM, Gruchala NE. Babies breaking bad: neonatal and iatrogenic withdrawal syndromes. Curr Opin Pediatr 2013; 25:532-42. https://doi.org/10.1097/MOP.0b013e328362cd0d.

[5] Harris J, Ramelet AS, van Dijk M, Pokorna P, Wielenga J, Tume L, et al. Clinical recommendations for pain, sedation, withdrawal and delirium assessment in critically ill infants and children: an ESPNIC position statement for healthcare professionals. Intensive Care Med 2016;42:972-86. https:// doi.org/10.1007/s00134-016-4344-1.

[6] Anand KJS, Willson DF, Berger J, Harrison R, Meert KL, Zimmerman J, et al. Tolerance and withdrawal from prolonged opioid use in critically III children. Pediatrics 2010; 125. https://doi.org/10.1542/peds.2009-0489.

[7] Duceppe MA, Perreault MM, Frenette AJ, Burry LD, Rico P, Lavoie A, et al. Frequency, risk factors and symptomatology of iatrogenic withdrawal from opioids and benzodiazepines in critically Ill neonates, children and adults: a systematic review of clinical studies. J Clin Pharm Therapeut 2019;44: 148-56. https://doi.org/10.1111/jcpt.12787.

[8] Ista E, Van Dijk M. Knowing risk factors for iatrogenic withdrawal syndrome in children may still leave us emptyhanded. Crit Care Med 2017;45:141-2. https://doi.org/ 10.1097/CCM.0000000000002160.

[9] Abou Elella R, Adalaty H, Koay YN, Mokrusova P, Theresa M, Male B, et al. The efficacy of the COMFORT score and pain management protocol in ventilated pediatric patients following cardiac surgery. Int J Pediatr Adolesc Med 2015;2:123-7. https://doi.org/10.1016/j.ijpam.2015.11.001.

[10] Best KM, Wypij D, Asaro LA, Curley MAQ. Patient, process, and system predictors of iatrogenic withdrawal syndrome in critically ill children. Crit Care Med 2017;45:e7-15. https:// doi.org/10.1097/CCM.0000000000001953.

[11] Best KM, Boullata JI, Curley MAQ. Risk factors associated with iatrogenic opioid and benzodiazepine withdrawal in critically ill pediatric patients: a systematic review and conceptual model. Pediatr Crit Care Med 2015;16:175-83. https://doi.org/10.1097/PCC.0000000000000306.

[12] Amirnovin R, Sanchez-Pinto LN, Okuhara C, Lieu P, Koh JY, Rodgers JW, et al. Implementation of a risk-stratified opioid and benzodiazepine weaning protocol in a pediatric cardiac ICU. Pediatr Crit Care Med 2018;19:1024-32. https://doi.org/ 10.1097/PCC.0000000000001719.

[13] Johnson PN, Boyles KA, Miller JL. Selection of the initial methadone regimen for the management of iatrogenic opioid abstinence syndrome in critically ill children. Pharmacotherapy 2012;32:148-57. https://doi.org/10.1002/ PHAR.1001.

[14] Oschman A, McCabe T, Kuhn RJ. Dexmedetomidine for opioid and benzodiazepine withdrawal in pediatric patients. Am J Health Pharm 2011;68:1233-8. https://doi.org/10.2146/ ajhp100257.

[15] Ista E, De Hoog M, Tibboel D, Duivenvoorden HJ, Van Dijk M. Psychometric evaluation of the sophia observation withdrawal symptoms scale in critically ill children. Pediatr Crit Care Med 2013;14:761-9. https://doi.org/10.1097/ PCC.0b013e31829f5be1.

[16] Nakayama Y, Shibasaki M, Shime N, Nakajima Y, Mizobe T, Sawa T. The RACHS-1 risk category can be a predictor of perioperative recovery in Asian pediatric cardiac surgery patients. J Anesth 2013;27:850-4. https://doi.org/10.1007/ s00540-013-1645-1.

[17] Larsen SH, Pedersen J, Jacobsen J, Johnsen SP, Hansen OK, Hjortdal V. The RACHS-1 risk categories reflect mortality and length of stay in a Danish population of children operated for congenital heart disease. Eur J Cardio-Thorac Surg 2005;28:877-81. https://doi.org/10.1016/j.ejcts.2005.09.008.

[18] Boethig D, Jenkins KJ, Hecker H, Thies WR, Breymann T. The RACHS-1 risk categories reflect mortality and length of hospital stay in a large German pediatric cardiac surgery population. Eur J Cardio Thorac Surg 2004;26:12-7. https:// doi.org/10.1016/j.ejcts.2004.03.039.

[19] Ávila-Alzate JA, Gómez-Salgado J, Romero-Martín M, Martínez-Isasi S, Navarro-Abal Y, Fernández-García D. Assessment and treatment of the withdrawal syndrome in paediatric intensive care units: systematic review. Medicine (Baltim) 2020;99:e18502. https://doi.org/10.1097/MD.000000 0000018502 . 